\title{
Ultrafast geometric manipulation of electron spin and detection of the geometric phase via Faraday rotation spectroscopy
}

\author{
Xin-Qi Li, Cheng-Yong Hu, Li-Xiang Cen, and Hou-Zhi Zheng \\ National Laboratory for Superlattices and Microstructures, Institute of Semiconductors, Chinese Academy of Sciences, P.O. Box 912, \\ Beijing 100083, China \\ YiJing Yan \\ Department of Chemistry, Hong Kong University of Science and Technology, Kowloon, Hong Kong, China
}

(Received 28 June 2002; published 27 December 2002)

\begin{abstract}
Time-resolved Faraday rotation spectroscopy is currently exploited as a powerful technique to probe spin dynamics in semiconductors. We propose here an all-optical approach to geometrically manipulate electron spin and to detect the geometric phase by this type of extremely sensitive experiment. The global nature of the geometric phase can make the quantum manipulation more stable, which may find interesting applications in quantum devices.
\end{abstract}

DOI: 10.1103/PhysRevB.66.235207

PACS number(s): 78.47.+p, 03.65.Vf

Faraday rotation (FR) describes the phenomenon of the polarization of linearly polarized light, after transmitting through a magnetized medium, being rotated around the light propagation or wave-vector direction. FR results from the refraction index difference between the left- and rightcircularly polarized light, and the amount of FR is proportional to the sample magnetization along the light propagation direction. Therefore, FR can be exploited as a noninvasive and sensitive means to measure material magnetization. In addition, the extreme sensitivity of the technique allows one to develop an efficient method (i.e., the timeresolved pump-probe approach) to monitor and measure the spin dynamics of photoexcited electrons, holes, and excitons in semiconductors. 1,2

In this paper, we elaborate a scheme of ultrafast geometric manipulation of electron spin in semiconductors by an alloptical approach and detection of the geometric phase via FR spectroscopy. Geometric phases, either the adiabatic Berry phase $^{3}$ or the nonadiabatic Aharonov-Anandan (AA) phase, ${ }^{4}$ are of fundamental interest in physics. Their existence has been experimentally demonstrated in many physical systems, such as molecules, ${ }^{5}$ light polarization in fibers, ${ }^{6}$ NMR, ${ }^{7-9}$ and neutron polarization. ${ }^{10}$ In particular, also based on the FR principle, a scheme to determine the geometric phase (with a unique value $\pi$ ) was proposed, ${ }^{11}$ where the geometric phase is associated with the light polarization vector, and the FR turns out to be an optical analog of a spin-1/2 particle in a magnetic field. More recently, in the context of geometric quantum computation, ${ }^{12}$ both the conditional Berry and AA phases were measured in a liquid NMR system ${ }^{13,14}$ and were proposed to construct a quantum logic gate. This interest is rooted in the fact that quantum manipulation by geometric means seems to be fault tolerant to certain types of computational errors. We also notice that, with the advent of spintronics, there exists a rapidly growing interest in coherent control of spins in semiconductors. The spin degree of freedom with its coherent control in solid states is widely believed to have a sound potential in quantum device applications. We thus anticipate that the study of the geometric manipulation of electron spin and the detection of the geo- metric phase via the extremely sensitive FR spectroscopy would be relevant to this remarkable trend.

Figure 1 depicts the time-resolved pump-probe FR spectroscopic setup. ${ }^{1,2}$ Here, the pump laser pulse, which is circularly polarized, is applied first to create electron-hole pairs in the semiconductor sample. As a result of the selection rule imposed by angular momentum conservation, only electronhole pairs consistent with the photon polarization can be excited. Owing to the distinct relaxation time scales (the hole spin relaxation is typically much faster), the hole- and electron-spin relaxations are usually addressed separately. As a good approximation, in this work we only consider the effect of electron spins (in the conduction band). Conventionally, photoexcited carrier (conduction electron) spins are subjected to the action of a magnetic field, and the timeresolved FR signal reveals the motion of spins. In this work, we are interested in spin motion involving a geometric phase that in turn generates an observable effect in the FR signal. Viewing the typical electron-spin relaxation time of nanoseconds, ${ }^{1,2}$ magnetic field pulses shorter than this time scale are needed in order to implement the desired cyclic quantum evolution of the spin state. Unfortunately, such short magnetic field pulses are beyond the scope of current technology. Very recently, an all-optical approach to the ultrafast manipulation of electron spin has been demonstrated by making use of the optical ac Stark effect that produces ultrashort effective magnetic field pulses. ${ }^{2}$ In what follows, we first elaborate the principle of geometrically manipulating the electron spin and using FR to detect the geometric phase,

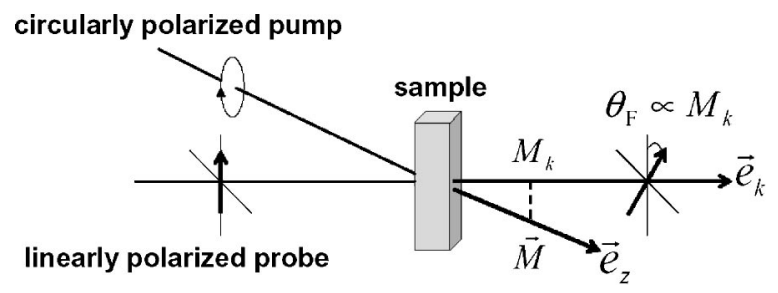

FIG. 1. Schematic diagram for the principle of Faraday rotation spectroscopy. 


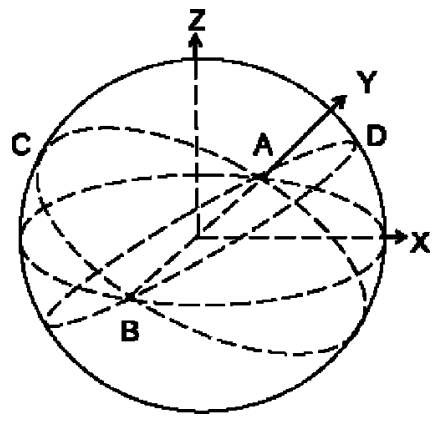

FIG. 2. Schematic diagram for geometric rotation of the state vector around the magnetic field. Since the state is always perpendicular to the field, there is no dynamical phase accumulation during the evolution.

then discuss the possible experimental implementation by an all-optical approach.

For the sake of generality, assume a mixed initial state of the carrier spins prepared by the pump laser in the conduction band (the hole spins in the valence band can be ignored because of their much shorter relaxation time):

$$
\rho_{i}=w_{0}|0\rangle\left\langle 0\left|+w_{1}\right| 1\right\rangle\langle 1| \text {. }
$$

Here, $|0\rangle$ and $|1\rangle$ denote, respectively, the spin-down and spin-up states of the excited electron, with respect to the pump laser direction along the $z$ axis (see Fig. 1). Correspondingly, we define the $x$ axis in the plane determined by the pump and probe laser propagating directions and the $y$ axis perpendicular to this plane. The time evolution of the density operator is governed by the Liouville equation, which results in a formal solution

$$
\rho(t)=U(t) \rho_{i} U^{\dagger}(t),
$$

where the operator $U(t)$ describes the quantum evolution starting from the initial (mixed) state $\rho_{i}$.

To evolve the electron-spin state, assume a magnetic field applied in the $x-z$ plane with, correspondingly, two projective components $B_{x}$ and $B_{z}$. For clarity, we first consider the nonadiabatic geometric evolution of the eigenstates of $\sigma_{y}$, $\sigma_{y}| \pm\rangle= \pm| \pm\rangle$, where $\sigma_{y}=\boldsymbol{\sigma} \cdot \boldsymbol{e}_{y}$ is the Pauli spin operator defined along the $\boldsymbol{e}_{y}$ direction ( $y$ axis). Specifically, quantum cyclic evolution is accomplished by the following magnetic field pulses: (i) Switching on a $\pi$ pulse of the magnetic field with components $B_{z}$ and $B_{x}$, the state $|+\rangle$ rotates around the magnetic field, from $|+\rangle$ in the $\boldsymbol{e}_{y}$ direction to $|-\rangle$ in the $-\boldsymbol{e}_{y}$ direction along curve $A C B$ on the Bloch sphere; see Fig. 2. (ii) Suddenly changing the magnetic field to another direction with components $B_{z}$ and $-B_{x}$, subjected to another $\pi$-pulse action the state $|-\rangle$ rotates back to $|+\rangle$ along curve $B D A$ on the Bloch sphere. From the AA phase theory, ${ }^{4}$ after the above cyclic evolution, the state $|+\rangle$ will acquire a geometric AA phase $e^{i \gamma}$, with $\gamma=4 \arctan \left(B_{x} / B_{z}\right)$. Note that during the above operation, the state vector is always perpendicular to the magnetic field; thus no dynamic phase is accumulated in the evolution. This idea was first promoted by
Suter et al. in their seminal experiment of demonstrating the AA phase. ${ }^{9}$ Similarly, the state $|-\rangle$ will acquire an AA phase $e^{-i \gamma}$ at the same time.

In analogy with the Mach-Zender interferometer where the photon wave function is split at the first beam splitter, we consider the consequence of the above rotation on the eigenstates of $\sigma_{z}: \quad|0\rangle=(-i / \sqrt{2})(|+\rangle-|-\rangle)$ and $|1\rangle=(1 /$ $\sqrt{2})(|+\rangle+|-\rangle)$. Straightforwardly, the quantum state evolution is described by

$$
\begin{aligned}
& |0\rangle \rightarrow \cos \gamma|0\rangle+\sin \gamma|1\rangle, \\
& |1\rangle \rightarrow \cos \gamma|1\rangle-\sin \gamma|0\rangle .
\end{aligned}
$$

We see here that the geometric AA phase plays a role of rotating $|0\rangle$ and $|1\rangle$ into their superposition states. As a consequence, geometric rotation of the electron spin is realized. As emphasized in the geometric quantum computation, ${ }^{12}$ the major advantage of geometric manipulation is its ability to be fault tolerant to certain types of errors. Note also that the possible value of $\gamma$ ranges from 0 to $2 \pi$ by controlling the magnetic field components $B_{x}$ and $B_{z}$, implying an arbitrary geometric rotation of the spin state.

Below we show that the geometric phase can be detected by FR. In the representation of the basis states $\{|1\rangle,|0\rangle\}$, the evolution described by Eq. (3) can be equivalently reexpressed in terms of the evolution operator (matrix) as

$$
U(T)=\left(\begin{array}{cc}
\cos \gamma & \sin \gamma \\
-\sin \gamma & \cos \gamma
\end{array}\right),
$$

where $T$ is the entire geometric rotation time. Substituting Eq. (4) into Eq. (2), the final state of the conduction electron spins after the above geometric operation reads

$$
\begin{aligned}
\rho_{f} & =U(T) \rho_{i} U^{\dagger}(T) \\
& =\left(\begin{array}{cc}
w_{1} \cos ^{2} \gamma+w_{0} \sin ^{2} \gamma & \left(w_{0}-w_{1}\right) \cos \gamma \sin \gamma \\
\left(w_{0}-w_{1}\right) \cos \gamma \sin \gamma & w_{1} \sin ^{2} \gamma+w_{0} \cos ^{2} \gamma
\end{array}\right) .
\end{aligned}
$$

With this result, any physical quantities can be carried out by statistically averaging the corresponding variables over the system density matrix. In the context of FR experiments, the relevant quantity is the projection of the sample magnetization in the propagating direction $\left(\boldsymbol{e}_{k}\right)$ of the probe light. The corresponding variable operator is $\sigma_{k}=\boldsymbol{\sigma} \cdot \boldsymbol{e}_{k}=\cos \alpha \sigma_{z}$ $-\sin \alpha \sigma_{x}$, where $\alpha$ is the angle between the pump- and probe-laser propagating directions. Straightforwardly, the component along $\boldsymbol{e}_{k}$ of the sample magnetization is calculated as

$$
M_{k} \propto \operatorname{Tr}\left(\sigma_{k} \rho_{f}\right)=\left(w_{1}-w_{0}\right) \cos (\alpha-2 \gamma) .
$$

Two valuable observations on Eq. (6) are in order: (i) The geometric phase $\gamma$ has a clear observable effect in $M_{k}$, thus in FR experiments. The dependence of the FR angle $\theta_{F}$ $\left(\propto M_{k}\right)$ on the geometric phase $\gamma$ is in terms of the typical behavior of quantum interferences. In the limit of $\gamma=0$, Eq. (6) reduces to the result of FR experiments that probes the initially excited carrier spins in the semiconductor sample. (ii) The output intensity is an incoherently weighted average 
of the pure-state interference profiles. It is easy to check that for pure states $|1\rangle$ and $|0\rangle$, the interference profiles are, respectively, $\pm \cos (\alpha-2 \gamma)$. The weight factors (i.e., $w_{1}$ and $w_{0}$ ) are given in the initial state $\rho_{i}$. This structure is consistent with the recent work on geometric phases for mixed states in interferometry. ${ }^{15}$

The interferometry described above is also applicable to detect the adiabatic Berry phase, ${ }^{3}$ provided the magnetic field can adiabatically complete a closed path in parameter space within the spin relaxation time. This condition can be satisfied in some spin systems, such as liquid NMR or doped spins in solid states. Particularly, if the initial state is an eigenstate of the system Hamiltonian, the adiabaticity will ensure that the state in the subsequent time will remain in the same eigenstate of the instantaneous Hamiltonian $H(\boldsymbol{B})(\boldsymbol{B}$ is the time-dependent magnetic field). As $\boldsymbol{B}$ traces a closed loop in the parameter space, a geometric phase is acquired. For the geometric-phase-based interference study, the initial state $|0\rangle$ (or $|1\rangle$ ) is a linear superposition of the eigenstates $| \pm\rangle$ of $H(\boldsymbol{B})$, if the magnetic field is initially along the $y$ direction. As a result of adiabatically dragging the Hamiltonian along a closed loop, a phase difference between $|+\rangle$ and $|-\rangle$ is caused. Noticeably, the phases acquired in this way have both geometric and dynamic contributions. To detect merely the geometric Berry phase via an interferometry, it is necessary to eliminate the dynamic phase. An applicable approach is to use the refocusing technique known as spin echo developed in NMR experiment, ${ }^{16}$ which was also employed recently to eliminate the dynamic phases in geometric quantum computations. ${ }^{13}$ The basic idea is to apply the cyclic evolution twice, with the second cyclic evolution retracing the first one but following the reversed path, and with the second application surrounded by a pair of fast $\pi$ transformations that swap the states $|+\rangle$ and $|-\rangle$. The net effect of this compound operation is to cancel the dynamic phases and maintain the geometric ones. Supposing that the net geometric phases acquired by $| \pm\rangle$ are $e^{ \pm i \gamma_{B}}$, the interference pattern is analogous to the previous nonadiabatic one, only with $\gamma$ in Eq. (6) being replaced by $\gamma_{B}$.

Now let us return to the possible experimental implementation of the ultrafast geometric manipulation of electron spin and the detection of the nonadiabatic AA phase, which is the major concern of the present study. As we have mentioned earlier, a necessary condition for realizing the geometric manipulation is the cyclic evolution being much faster than the spin relaxation. Noting that the conduction-electron spin relaxation time is about tens of nanoseconds, we thus need ultrashort magnetic field pulses. We show below how this goal can be achieved by virtue of a recent experiment on the ultrafast manipulation of electron spin by a novel alloptical approach. ${ }^{2}$

The basic idea is to make use of an off-resonance ultrafast laser pulse to induce ac Stark shifts, which are in turn equivalent to the result of an effective magnetic field. To illustrate this, consider the energy level diagram of a semiconductor quantum well shown in Fig. 3(a). In the absence of a magnetic field, the lowest conduction-band (CB) level is twofold degenerate, denoted by spin states $| \pm 1 / 2\rangle$, and the valence-band (VB) states are denoted, respectively, by (a)

(b)

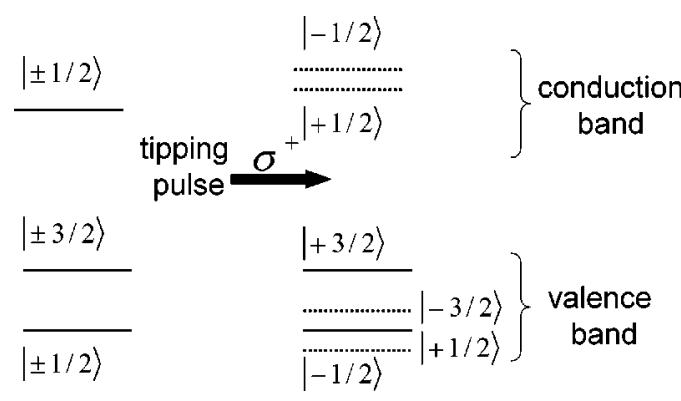

FIG. 3. ac Stark shifts induced by an off-resonance below-bandgap laser pulse. Depicted are, respectively, the original unperturbed energy levels (a) and the shifted ones (b).

$| \pm 3 / 2\rangle$ and $| \pm 1 / 2\rangle$. Switching on a below-band-gap laser pulse (tipping pulse) with, for example, $\sigma^{+}$polarization, the laser will virtually couple the state pairs between the VB state $|-3 / 2\rangle$ and CB state $|-1 / 2\rangle$, as well as the CB state $|+/ 2\rangle$ and VB state $|-1 / 2\rangle$. Other possible couplings between laser and electron-hole pairs are forbidden from the standpoint of angular momentum conservation. It is well known that this kind of off-resonance coupling will cause ac Stark shifts of the relevant energy levels. In particular, the energy shifts can be estimated from second-order perturbation theory as follows: $\Delta E_{-1 / 2}^{c}=\left|V_{-3 / 2,-1 / 2}\right|^{2} / \Delta_{1}$ for the CB state $|-1 / 2\rangle, \Delta E_{-3 / 2}^{v}=\left|V_{-3 / 2,-1 / 2}\right|^{2} /\left(-\Delta_{1}\right)$ for the VB state $|-3 / 2\rangle, \Delta E_{1 / 2}^{c}=\left|V_{-1 / 2,+1 / 2}\right|^{2} / \Delta_{2}$ for the CB state $|+1 / 2\rangle$, and $\Delta E_{-1 / 2}^{v}=\left|V_{-1 / 2,+1 / 2}\right|^{2} /\left(-\Delta_{2}\right)$ for the VB state $|-1 / 2\rangle$. Here, $\Delta_{1}$ and $\Delta_{2}$ are the detunings of the photon energy with the two pair states; $V_{-3 / 2,-1 / 2}$ and $V_{-1 / 2,+1 / 2}$ are coupling matrix elements of the laser with the state pairs. Plotted in Fig. 3(b) are the shifted levels, with respect to the original unperturbed ones in Fig. 3(a). We notice here that the level repulsive effect is obvious.

As experimental studies have indicated that the hole spins are either pinned along the quantum well growth direction or dephase rapidly, ${ }^{1,2}$ the FR dominantly measures the net effect of the $\mathrm{CB}$ electron spins. As a consequence, the ac Stark shifts of CB states $|-1 / 2\rangle$ and $|+1 / 2\rangle$ can be equivalently described as the effect of an effective magnetic field $B_{\text {eff }}$, along the tipping-pulse direction. Denoting the $C B$ level splitting by $\delta_{\mathrm{CB}}$, the effective magnetic field $B_{\text {eff }}$ $=\delta_{\mathrm{CB}} /\left(g_{c} \mu_{B}\right)$, where $g_{c}$ is the Lande $g$ factor and $\mu_{B}$ is the Bohr magneton. As a rough estimate, corresponding to a $\mathrm{CB}$ level splitting of $\delta_{\mathrm{CB}} \sim 1 \mathrm{meV}$, the effective magnetic field $B_{\text {eff }}$ can be as high as $\sim 20 \mathrm{~T}$. In addition, the persistence time of this effective magnetic field is identical to the laserpulse duration time, which can be as short as femtoseconds. Therefore, the resulting effective magnetic field can suffice for the geometric operation (rotation) of the electron spin under study. To obtain the interference pattern originated from the AA phase, the FR experiment can be arranged as follows: (i) After optical excitation using an ultrafast circularly polarized laser pulse, the initial spin state is prepared along the $z$ direction shown in Fig. 1. In the absence of a magnetic field, the FR angle of the linearly polarized probe 
laser remains unchanged. (ii) Switch on two tipping pulses in succession such that the effective magnetic field induced by the ac Stark effect rotates the electron spin in the manner as shown in Fig. 2. Note that the direction of the effective magnetic field is given by the tipping-pulse propagating direction, and its magnitude depends on the detunings and coupling strengths between the tipping laser and electron-hole pairs. After the action of the tipping pulses, the FR angle will gain a change as show by Eq. (6). (iii) Repeat procedures (i) and (ii) by making changes of the tipping-pulse directions. A series of values of the AA phase $\gamma$ can be measured, and accordingly the interference pattern resulting from the geometric AA phase can be obtained.

In summary, we have elaborated an experimentally accessible scheme to geometrically manipulate electron spin and to detect the nonadiabatic geometric phase via FR spectroscopy. This study might be of fundamental interest and rel- evant to the notion of spintronics. Viewing the particular significance of ultrafast manipulation of electron spin, the proposed study can further confirm the novel approach developed in Ref. 2. Moreover, the proposed geometric scheme may further stabilize the quantum operation, i.e., being of fault tolerance to some operational errors. Finally, the proposed interference scheme based on the FR setup can also be applied to detect geometric phases in other spin systems, including the adiabatic Berry phase, as long as the spin relaxation time is longer than the experimentally accessible magnetic field duration time.

Support from the Major State Basic Research Project No. G001CB3095 of China, the Special Fund for "100 Person Project" from the Chinese Academy of Sciences, and the Research Grants Council of the Hong Kong Government are gratefully acknowledged.
${ }^{1}$ S.A. Crooker, D.D. Awschalom, and N. Samarth, IEEE J. Select. Topics Quantum Electron. 1, 1082 (1995); S.A. Crooker, D.D. Awschalom, J.J. Baumberg, F. Flack, and N. Samarth, Phys. Rev. B 56, 7574 (1997).

${ }^{2}$ J.A. Gupta, R. Knobel, N. Samarth, and D.D. Awschalom, Science 292, 2458 (2001).

${ }^{3}$ M.V. Berry, Proc. R. Soc. London, Ser. A 392, 45 (1984).

${ }^{4}$ Y. Aharonov and J. Anandan, Phys. Rev. Lett. 58, 1593 (1987).

${ }^{5}$ G. Delacretaz et al., Phys. Rev. Lett. 56, 2598 (1986).

${ }^{6}$ R.Y. Chiao and Y.S. Wu, Phys. Rev. Lett. 57, 933 (1986); T. Tomita and R.Y. Chiao, ibid. 57, 937 (1986).

${ }^{7}$ R. Tycko, Phys. Rev. Lett. 58, 2281 (1987).

${ }^{8}$ D. Suter et al., Mol. Phys. 61, 1327 (1987).

${ }^{9}$ D. Suter, K.T. Mueller, and A. Pines, Phys. Rev. Lett. 60, 1218
(1988).

${ }^{10}$ T. Bitter and D. Dubbers, Phys. Rev. Lett. 59, 251 (1987).

${ }^{11}$ J. Zak, Phys. Lett. A 154, 471 (1991).

${ }^{12}$ P. Zanardi and M. Rasetti, Phys. Lett. A 264, 94 (1999); J. Pachos, P. Zanardi, and M. Rasetti, Phys. Rev. A 61, 010305(R) (2000).

${ }^{13}$ J.A. Jones, V. Vedral, A. Ekert, and G. Castagnoli, Nature (London) 403, 869 (2000).

${ }^{14}$ J. Du, M. Shi, J. Wu, X. Zhou, and R. Han, quant-ph/0207022 (unpublished).

${ }^{15}$ E. Sjöqvist, A.K. Pati, A. Ekert, J.S. Anandan, M. Ericsson, D.K.L. Oi, and V. Vedral, Phys. Rev. Lett. 85, 2845 (2000).

${ }^{16}$ R.R. Ernst, G. Bodenhausen, and A. Wokaun, Principles of Nuclear Magnetic Resonance in One and Two Dimensions (Clarendon Press, Oxford, 1987). 